\title{
HACIA UNA TEORÍA DE LOS CONTENIDOS ESCOLARES*
}

\section{Izquierdo AyMerich, Mercè}

Departament de Didàctica de les Ciències. Universitat Autònoma de Barcelona

\begin{abstract}
Resumen. En esta conferencia se reflexiona sobre los temas de ciencias que se estudian en la escuela y sobre el significado del término contenidos, que se utiliza a menudo cuando los profesores se refieren a ellos. Las nuevas aportaciones de las ciencias cognitivas y de la lingüística muestran que «conocer» requiere «actividad» y sugieren que los «contenidos escolares» han de hacer posible el desarrollo de actividad científica de la cual los alumnos sean los protagonistas.

Como consecuencia de la reflexión se identifican algunas de las condiciones que deberían cumplir los programas de ciencias y se propone un itinerario para desarrollar los temas de las ciencias en la escuela.
\end{abstract}

Palabras clave. Didáctica de las ciencias, contenidos, ciencia para todos.

Summary. This paper deals with the subjects on science studied at school and with the meaning of the term «content», often used by teachers to refer to them. New contributions by cognitive science and linguistics show that «knowing» requires «activity» and hint that «school content» must enable the development of the scientific activity carried out by the learners.

As a result from this reflection we identify some of the requirements that science programmes should comply with and propose an itinerary to develop science subjects at school.

Keywords. Didactics of science, content, science available to everybody.

\section{¿QUÉ ENSEÑAR? ÉSTE ES EL PROBLEMA}

Uno de los problemas propios de la didáctica de las ciencias (DC) es cómo seleccionar adecuadamente lo que se va a enseñar, para que los alumnos puedan aprenderlo ${ }^{1}$. Aceptar que esto es un problema significa aceptar también que los «contenidos» de la enseñanza no están prefijados y que pueden cambiarse en función de los objetivos de aprendizaje que se hayan establecido (Rué, 2002).

No es fácil reconocer que esto es un problema. La tendencia general es suponer que lo que sabemos (o cree- mos que aprendimos los que ahora somos adultos) es lo que todos han de saber. Puesto que cuesta aceptar que quizás nada de ello (o casi nada) interesa o es necesario ahora, se tiende a pensar que la didáctica puede «maquillar» cualquier tema y que es suficiente cambiar la fachada de los temas y la manera de enseñarlos para que los alumnos los aprendan. En consecuencia, desaparece el problema y la didáctica queda relegada a una metodología, perdiendo así su capacidad de fundamentar los nuevos currículos que está necesitando la enseñanza de las ciencias. 
Pero hay indicios claros de que este problema existe, dentro y fuera de la escuela. En la escuela, se deduce de la concepción sistémica de la clase; y fuera de la escuela, lo plantean la proliferación de nuevos currículos, los fracasos escolares y la oferta de conocimientos de la nuevas tecnologías de la información y la comunicación (NTIC) .

\section{Los contenidos, uno de los elementos del sistema didáctico}

Chevellard (1991) se ha referido a la clase como a un sistema didáctico, cuyos elementos son los alumnos, los profesores y los contenidos; como en todos los sistemas, estos elementos interaccionan y la interación óptima se produce cuando la acción docente es eficaz y los alumnos aprenden (Fig. 1).

Figura 1

La didáctica de las ciencias es una manera de mirar los contenidos de ciencias desde la perspectiva de tener que enseñarlas.

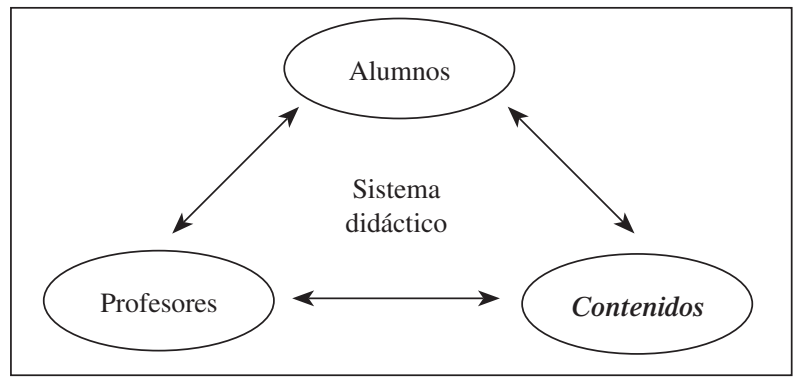

Considerar seriamente que los contenidos están en función de los otros elementos del sistema significa que se tiene en cuenta una nueva cualidad del conocimiento: que sea «moldeable» para que puedan aprenderlo unos alumnos concretos, ya no todo puede ser aprendido significativamente ni de la misma manera por todas las personas. La profesionalidad del docente se manifiesta en la capacidad de organizar un escenario en el cual se aprenda la ciencia que se enseña determinada con una finalidad educativa, mediante un complejo proceso de «transposición» o «reconstrucción» didáctica (Gundem, 2000). (No se trata de continuar con aquello de que «la letra con sangre entra» ni pensar que la DC puede ofrecer recursos para «tragarse» cualquier cosa).

Desde esa perspectiva, la didáctica de las ciencias se puede considerar «una manera de mirar» el conocimiento científico al tenerlo que comunicar tanto a personas expertas como no expertas, de tal manera que contribuya a su desarrollo personal. «Los contenidos» pasan a ser una variable respecto a la cual se han de tomar decisiones; es decir, un «problema» para la DC.

\section{Debate social sobre los contenidos}

La escuela y la universidad están siendo objeto de un debate público en los últimos años y el «problema de los contenidos» se plantea de manera diversa y contra- dictoria. Se critica que «bajan los contenidos», aunque muchos creen que los conocimientos enciclopédicos no son útiles. Los profesores son criticados tanto porque no enseñan «lo de siempre» como porque no explican los conocimientos más recientes ni ciencia aplicable a la vida; y las disciplinas científicas clásicas captan cada vez menos alumnos.

Estas críticas presentan diferentes dimensiones: social, humanista, económica, política, prospectiva... que nos hacen ver que reflexionar sobre los contenidos de la enseñanza también tiene sentido fuera de la escuela.

a) Dimensión social: los conocimientos han de ser útiles y asequibles a toda la población. Se reclama alfabetización científica, «ciencia para todos» y competencias básicas pero también especialización y competencias de alto nivel.

b) Dimensión humanista: las ciencias son para las personas y están sujetas a valores humanos. No han de ser «cientistas»; se ha de priorizar la «sabiduría» (que es conocimiento que se saborea y que hace feliz) y rechazar el enciclopedismo considerado, ahora, estéril.

c) Dimensión económica: las nuevas tecnologías permiten vender los conocimientos científicos en nuevos formatos y aparece un público que «compra» software, libros, películas, revistas de divulgación científica y que asiste a museos y conferencias. Pero no se puede engañar al público: la información se puede comprar, pero no el conocimiento.

d) Dimensión política: se está configurando un nuevo espacio educativo europeo que requiere tanto homologar conocimientos como flexibilizarlos. Se busca la excelencia (¿de unos cuántos?) pero también respetar la diversidad cultural.

e) Dimensión de futuro o el síndrome «siglo XXI»: ¡no puede ser que estemos explicando lo mismo que hace cien años, y casi de la misma manera! Las nuevas tecnologías han de hacer mucho más que poner los documentos escritos de siempre en internet.

Probablemente estamos viviendo una etapa de cambio, en la que se deja atrás la época del «conocimiento especializado» (como ésta superó el «conocimiento razonado» de la Ilustración) y se avanza hacia una sociedad del conocimiento en la cual todas las personas puedan participar en sistemas didácticos generadores de conocimientos aplicables, adecuados a sus expectativas.

Si esto fuera así, los «conocimientos» no estarían «contenidos» en ningún «contenedor» (sea libro, museo, CD o vídeo); serían «algo» que no sólo «se sabe» sino que permite actuar con criterio.

¿Podría ser que estuviéramos iniciando la época del «conocimiento sabio», dejando atrás la del «conocimiento enciclopédico»? Sería así si los temas que se enseñan se 
seleccionan y modelan pensando en las capacidades de los alumnos y no sólo en las disciplinas.

\section{PERTINENCIA DE LA DIDÁCTICA DE LAS CIENCIAS PARA RESOLVER ESTE PROBLEMA}

Volvamos a la escuela, que es el espacio de intervención y reflexión de los que nos ocupamos de la enseñanza de las ciencias ${ }^{2}$; y con ello, a la figura 1 . Si, como hemos dicho, partimos de considerar el aula de clase como un sistema didáctico (SD), la «ciencia» no está en el libro o en la lección, sino en lo que el libro o la lección permiten pensar, hacer, comunicar a aquéllos que lo leen o la escuchan.

Es oportuno y pertinente que la DC desarrolle criterios teóricos para la selección y organización de unos conocimientos escolares que puedan funcionar en un SD y según las expectativas sociales y didácticas que se han ido exponiendo en el apartado anterior.

White (1994), en el libro The content of Science del cual fue editor, propone que una de las finalidades de la investigación en Science Education sea «una teoría de los contenidos escolares». En 199933, Fensham comentaba que este libro, con excepción del capítulo de White, había dejado el tema sin resolver, limitándose a comentar las «ideas previas» en las diferentes áreas del currículo. Al hacer su propia aportación al tema en una de las conferencias plenarias, Fensham introdujo una consideración de gran interés para nosotros. Se refirió a la diferencia entre la tradición de la Europa Continental de «didáctica de las ciencias (DC)» (que es la nuestra), en la que se da una gran importancia a los aspectos pedagógicos del contenido científico, y la anglosajona, «Science Education», en la cual se centra la atención en las estrategias docentes, y el profesorado se forma para un currículo del cual no es directamente responsable. Citando el libro de Klafki (1958), con el que se han formado los profesores alemanes durante un cuarto de siglo, selecciona las cinco preguntas que propone para un análisis didáctico de los contenidos.

1) ¿De qué profundo o general sentido de la realidad es ejemplo?

¿Qué fenómeno o principio fundamental, ley, criterio, problema, método, técnica o actitud se puede captar utilizándolo como ejemplo?

2) ¿Qué significado previo puede tener este concepto para el alumnado de mi clase?

¿Qué significado puede tener desde un punto de vista pedagógico?

3) ¿Qué aporta para el futuro de mis alumnos?

4) ¿Como se estructura pedagógicamente?

$5 a)$ ¿Qué hechos, fenómenos, situaciones, experimentos, controversias, intuiciones... son apropiados para inducir al alumnado a plantear preguntas dirigidas a la esencia y estructura del contenido en cuestión?

b) ¿Que imágenes, indicaciones, relatos, situaciones, observaciones, experimentos, modelos... son apropiados para ayudar al alumnado a responder de la manera más autónoma posible las preguntas dirigidas a los aspectos esenciales del tema?

c) ¿Qué situaciones y tareas son apropiadas para ayudar al alumnado a captar lo principal del tema mediante un ejemplo o un caso elemental, y para aplicarlo y practicarlo de manera que le resulte útil?

Si bien las preguntas 4 y 5 son comunes a ambas tradiciones, las tres primeras pertenecen sólo a la tradición didáctica, en la cual el profesor es responsable de su asignatura y son coherentes con la propuesta de «transposición didáctica», de Chevallard (1991) y Shulman (1995).

Según Fensham (2001), la investigación en ambas tradiciones aporta nuevas ideas que son relevantes para formular con precisión el problema e indica cómo avanzar en el análisis didáctico de los currículos. Son las siguientes:

- La importancia de la dimensión afectiva en la enseñanza y de la motivación obliga a ser muy cuidadosos en la presentación de los objetivos, a «negociarlos» y a avanzar hacia una educación científica «intercultural» que no excluya a nadie.

- Interesan nuevos temas, como lo muestra la aparición de currículos que enfatizan un determinado contenido para conseguir una determinada finalidad. Por ejemplo, Nature of Science, Solid Foundation, Self as explainer, Scientific Skills, Science / Technology decisions...

- Hasta ahora la investigación en «ideas previas» se ha centrado en los currículos «académicos»; a partir de ahora debería conceder más atención a las ideas científicas «populares» que correspondan a los nuevos temas emergentes (agujero de ozono, crisis energética...).

- Puesto que los «procedimientos» no son independientes de los contenidos y éstos van a ser diversos, no es posible enseñar según un «método científico» basado en la experimentación. Se han de utilizar las habilidades superiores de «razonamiento científico» enseñando a argumentar científicamente (Millar y Driver, 1987) para participar en un discurso científico (Kuhn, 1993; Ohlsson, 1995).

- Por todo ello, el profesorado necesita conocimiento metadisciplinar para estructurar los conocimientos (García, 1998; Izquierdo et al., 1999), que es necesario para estructurar una determinada propuesta, la cual va a ser transdisciplinar.

\section{Aportaciones recientes a la reflexión sobre los con- tenidos escolares}

Aportaciones teóricas diversas (Lemke, Vigotsky, Wittgenstein, Guidoni... ) a las que me referiré más adelante 
ayudan a comprender que ningún conocimiento científico es «un retrato del mundo», sino que siempre es el resultado de una intervención intencionada en determinados fenómenos, que ha sido interpretada y comunicada a personas que comparten valores y expectativas. Por ello, los conocimientos escolares no pueden ser ya «los programas» de siempre basados en conceptos que proporcionan una visión rígida y dogmática del mundo sino que han de ser la ocasión de plantear preguntas que inciten a la intervención y a la reflexión sobre fenómenos y relaciones entre fenómenos que sean relevantes para comprender los temas científicos de actualidad.

La diversidad de currículos que van apareciendo (dedicados a nuevas temáticas, con nuevos énfasis) sin que por ello cambie la escuela, la cual continua aferrada a libros de texto enciclopédicos (aunque mejor ilustrados y más atractivos), invita a una reflexión muy seria.

En España, García Díaz ha sido pionero en plantear abiertamente la necesidad de nuevos contenidos para actuar en coherencia con las aportaciones de la DC y a las nuevas necesidades educativas. Creo que su propuesta, en la que los contenidos han de articularse alrededor de «ideas estructurantes» con una finalidad de cambio social a través de la educación ambiental, puede aplicarse a otras finalidades y es, por lo tanto, una concreción de una «teoría de los contenidos escolares» más general. Soy deudora de esta aportación y la he tenido muy en cuenta.

Otros grupos están trabajando también en propuestas novedosas: nuevos currículos CTS, proyectos de ciencia integrada o coordinada... (De Pro y Saura, 2001). El título del número 29 de la revista Alambique (octubre 2001), «Nuevos tiempos, nuevos contenidos», es bien explícito y manifiesta la actualidad del tema y la necesidad de establecer algunos criterios que ayuden a consolidar las nuevas propuestas.

También en nuestro departamento hemos desarrollado una nueva propuesta de «ciencia escolar» fundamentada en un modelo cognitivo de ciencia ${ }^{4}$. (Izquierdo et al., 1999).

La DC podría proporcionar criterios para seleccionar conocimientos relevantes y educativos y valorar los nuevos currículo de «ciencia para todos» frente a los tradicionales de «ciencia de los conocimientos probados», teniendo en cuenta que:

- las respuestas afectivas a los contenidos condicionan el aprendizaje;

- el lenguaje para sustentar el razonamiento científico es diferente del lenguaje científico de los textos;

- no hay delimitación clara en los conocimientos disciplinares que forman parte de un currículo; la hay estratégica, en función de la finalidad educativa.

Con todo ello, aumenta la diferencia entre los temas escolares y los académicos. ¿Con qué recursos teóricos contamos para diseñar con criterio nuevos contenidos de ciencia en la escuela?

\section{BASE TEÓRICA: LA REVOLUCIÓN COGNITI- VA, UNA NUEVA FILOSOFÍA DEL LENGUAJE, UN NUEVO MODELO DE CIENCIA}

«Conocer» es el resultado de una interacción en la que intervienen los sentimientos, que transforma (al profesorado, al alumnado.... iy al conocimiento!) y que, en el contexto escolar, ha de ser educativa. Tiene consecuencias porque permite llevar a cabo actuaciones nuevas y tomar decisiones.

Los contenidos conocimientos científicos que se enseñan en la escuela han de generar experiencia y actividad, en un marco de valores. Requieren de una transposición (Chevallard, 1991) o de un análisis didáctico (Klafki, 1958; Hopmann y Riquart, 1995) que puede llegar a ser mucho más radical de lo que ha sido hasta ahora, puesto que el grupo humano al cual se ofrecen es muy diferente a aquél en el cual se generaron los conocimientos disciplinares.

Es necesario comprender mejor los factores que contribuyen a la emergencia de los conocimientos en un contexto determinado y a que éstos se puedan comunicar de manera adecuada a unos estudiantes concretos. Las ciencias cognitivas, la lingüística y la filosofía de la ciencia nos permiten iniciar esta reflexión.

\section{La revolución cognitiva: el cerebro-mente}

Hasta hace poco la psicología se debatía entre dos propuestas en las que «lo que hay en la mente» era, voluntariamente, una «caja negra»: el conductismo (Skinner, 1968) y las pruebas de inteligencia (cociente intelectual), inventadas por Binet en París, hace casi un siglo (Gardner et al., 1996). Según la primera, se puede aprender siempre y cuando la secuencia de actividades sea adecuada, con los mecanismos de premio/castigo más oportunos; según la otra, sólo aprenden los que son inteligentes... y los inteligentes son aquéllos que aprenden... porque han superado unas pruebas o tests normalizados.

A mediados de la década de los cincuenta se produce la «revolución cognitiva», que introduce el nuevo concepto de representación mental: las personas tienen ideas, imágenes y diversos lenguajes en su cerebro-mente, que son importantes y pueden ser estudiadas y modificadas. (¡Filósofos como Platón, Aristóteles, Descartes o Kant habían creído siempre en estas representaciones mentales!)

La ciencia cognitiva se interesa por la formación del conocimiento humano y convergen en ella ciencias muy diversas; muestra que es posible generar conocimiento, pero no de todo ni de cualquier manera. Y, más allá de la propia cognición, destaca la importancia de la motivación y de la capacidad de tomar decisiones en el proceso de emergencia de conocimiento.

Con la revolución cognitiva (Gardner, 2000, pp. 79-89), la mente deja de ser una «caja negra» y se introducen ideas que permiten nuevos planteamientos docentes. 
- La perspectiva del desarrollo cognitivo a lo largo de los primeros años de la vida (de Piaget) que condiciona la adquisición de conocimientos.

- La posibilidad de representaciones mentales universales. Chomsky (1980) reconoce que el lenguaje «tiene su propio sistema de representaciones psicológicas y (es de suponer) neurológicas» con las que se nace. Se identifican también otras estructuras mentales dedicadas a la conciencia del número, las relaciones espaciales, la música, la comprensión de otras personas... que son comunes a todos los seres humanos normales. Las pautas de uso de las representaciones no forman parte de las propias representaciones sino que dependen del contexto cultural; las actividades educativas requieren la interacción de representaciones diferentes, que no se pueden dejar al azar.

- La configuración de la personalidad. Según Gardner, hay diferentes «patrones de inteligencia» (lógicomatemática, espacial, musical, corporal-cinestésica, intrapersonal, interpersonal, existencial..), que se desarrollan y se relacionan entre ellas según las experiencias vividas. Por ello es necesario favorecer las interacciones y sería deseable enseñar lo más personificadamente posible.

- Se identifican algunas funciones cognitivas superiores. Algunas de ellas inciden en el «razonamiento científico»: resolución de problemas, planificación, creatividad, comprensión profunda... Otras permiten reflexionar sobre el proceso de «conocer»: la metamnemónica (sobre la propia memoria), metacognitiva (el propio pensamiento), de metarrepresentación (las propias representaciones).

- La cognición tiene que ver con la motivación, las emociones, la personalidad... La motivación aumenta cuando el aprendizaje es gratificante; requiere contacto con el adulto, actuación pública, practicar, hacer algo para lo que se está bien dotado. Las emociones indican qué temas y qué experiencias son agradables.

Una teoría de los contenidos escolares ha de tener en cuenta los puntos siguientes:

- El pensamiento trabaja en un espacio cognitivo que es multidimensional y «conocemos» cuando hay coincidencia entre diferentes «dimensiones».

- Son las vivencias lo que da sentido al conocimiento.

No hay una ruta directa que permita aplicar los descubrimientos neurocientíficos a la educación.

En resumen: parece ser que, en la escuela, se ha de hacer «trabajar como aprendices» pero potenciando las habilidades superiores. Es necesario un buen maestro que acompañe al aprendiz y cuyas acciones se puedan imitar, pero que deje un margen de libertad con el fin de ensayar las estrategias propias de cada cual para acceder al conocimiento.

\section{El lenguaje y la cognición en el contexto}

Guidoni (1985), también según la tradición cognitiva, se refiere a tres dimensiones cognitivas, irreductibles, que, al interaccionar, permiten la emergencia de conocimiento personal «en conexto»; son: el lenguaje, la representación mental y la acción. Sugiere que el conocimiento significativo se adquiere cuando hay coherencia entre las tres dimensiones y por ello permite hacer lo que se piensa y decirlo de tal manera que transforma tanto lo que se ha hecho como lo que se ha pensado... para poder actuar y pensar de nuevo (Fig. 2).

Figura 2

La interacción de las dimensiones cognitivas en el proceso de «conocer».

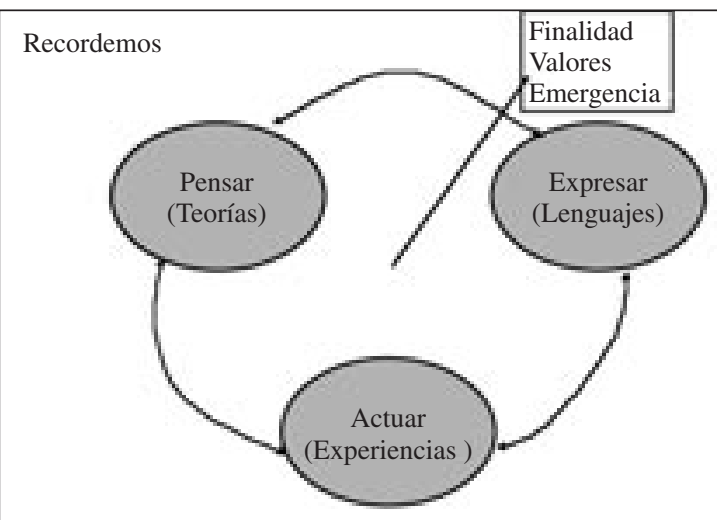

El mundo tira de nosotros

Ninguna de estas relaciones funciona fácilmente cuando, en la clase de ciencias, se han de introducir nuevos lenguajes (científicos) que se refieren a nuevas ideas (científicas).

En el «Discurs Preliminaire» del Traité de Chimie, de Lavoisier (1789), se propone una relación diferente entre conocimiento científico y lenguaje, que ha tenido (y continúa teniendo) una gran influencia entre los profesores y que ahora convendría revisar:

«Y en efecto, mientras que sólo creía ocuparme de la nomenclatura, mientras que mi único objeto era perfeccionar la lengua química, el trabajo se transformó insensiblemente en mis manos y, sin poderlo evitar, en un tratado elemental de química.

»La imposibilidad de aislar la nomenclatura de la ciencia y la ciencia de la nomenclatura, se debe a que toda ciencia física se forma necesariamente de tres cosas: la serie de hechos que constituye la ciencia, las ideas que los evocan y las palabras que los expresan. La palabra debe originar la idea, ésta debe pintar el hecho: he aquí tres huellas de un mismo cuño. Y como las palabras son las que conservan y transmiten las ideas, resulta que no se puede perfeccionar la lengua sin perfeccionar la ciencia, ni la ciencia sin la lengua; y por muy ciertos que fuesen los hechos, por muy justas que fuesen las ideas que origi- 
nasen, sólo transmitirían impresiones falsas si careciéramos de expresiones exactas para nombrarlas [...]

»[...]y yo he designado, cuando me ha sido posible, a las substancias simples con palabras sencillas y, por lo tanto, éstas son las primeras que me he visto obligado a nombrar [...] Respecto a los cuerpos formados por la unión de varias substancias simples, los hemos designado con nombres compuestos, por ser así ellos mismos; pero caeríamos en el desorden y confusión si no hubiésemos establecido clasificaciones[...]»

Como vemos, Lavoisier parece suponer que los hechos del mundo son los que generan, en el investigador, la idea científica y que es necesario disponer de la palabra más adecuada a la idea científica para poder hablar del hecho, que queda así interpretado y se hace comprensible.

\section{Hecho $\rightarrow$ Idea $\rightarrow$ Palabra}

Este esquema de Lavoisier ha sido utilizado en la enseñanza como si fuera reversible, como si pudiera funcionar en sentido inverso: la palabra científica genera en el estudiante la idea con la cual el hecho queda interpretado. Pero ha llegado el momento de poner en crisis este esquema, porque ahora sabemos que no puede evitarse hablar espontáneamente de los hechos con lenguajes que escapan al control científico; es decir, que la palabra se relaciona con el hecho de manera que escapan al control del profesorado.

En efecto, para nosotros, profesores de ciencias, lo más intrigante es la dificultad que aparece al interpretar la experimentación escolar mediante los términos que se refieren a entidades científicas. El profesorado «tiene» el término, que aparece en los libros de texto; pero el alumnado no tiene aún la idea científica. Entiende mejor «el agua disuelve la cal» (porque sabe de qué se está hablando) que «la disolución acuosa de dióxido de carbono está en equilibrio con ácido carbónico; por esto, reacciona con el carbonato de calcio y forma una sal ácida, el hidrogenocarbonato de calcio, que es soluble en agua», aunque esta última sea mucho más completa y precisa. Da la impresión de que la claridad comunicativa y el rigor sean incompatibles en la clase de ciencias (Lemke, 1997).

Para continuar esta reflexión sobre las relaciones entre el pensamiento, la acción y el lenguaje vamos a considerar que el lenguaje es el mediador entre las representaciones y las acciones que constituyen la experiencia científica: desde el punto de vista de lo experimental, el lenguaje cambia el formato del mundo para hacerlo pensable; desde el punto de vista de lo representacional, el lenguaje cambia el formato de las representaciones para hacerlas comunicables, en un determinado contexto, a una audiencia concreta. Aprovechando la figura 2, podemos decir que tanto las teorías como la experiencia científica son lo que son gracias al lenguaje, así como el lenguaje es lo que es porque es, a la vez, pensamiento y acción.

El conocimiento transmitido por el lenguaje no es totalmente objetivo (requiere acción, intervención personal) ni es totalmente subjetivo, sino que es «experiencial»y, por ello es, en gran parte, metafórico (Lakoff y Johnson 1980, p. 228-237).

Ésta es quizás la esencia del «problema de los contenidos» que ahora detectamos: que se había generado un concepto de contenido vinculado al lenguaje de los libros y no al «conocimiento», pensando (quizás) que el conocimiento está en las palabras y que, al implantarse las palabras (que puede hacerse), se implantan a la vez «experiencias» (que no puede hacerse). Por esto, es imprescindible considerar que, al referirnos al conocimiento, es imprescindible aludir a los aspectos comunicativos, sociales, del lenguaje con el cual se comunica. Si la praxis no corresponde a lo que un determinado lenguaje quiere expresar, el lenguaje no adquiere su pleno significado. Y, una vez contemplado el problema desde esta perspectiva, tenemos ya un camino hacia su resolución: se trata de diferenciar entre el conocimiento (que es multidimensional) y lo que se dice y escribe de él en un lenguaje concreto.

Nos dice Wittgenstein $(1997,1958)$ que surgen nuevos tipos de lenguaje y que otros son olvidados, porque «hablar un lenguaje» es una parte de la actividad o de una forma de vida; y la expresión «el juego del lenguaje» que él utiliza destaca este aspecto. El lenguaje propio de un «juego de lenguaje» no tiene significado fuera de él. Comprender el lenguaje de las ciencias (o cualquier otro lenguaje con el que se comunica conocimiento) requiere «jugar el juego» apropiado.

Debemos llegar a la conclusión de que sólo va a ser válido un programa de los conocimientos escolares que planifique a la vez lo teórico, lo experimental y lo lingüístico (lo que puede ser pensado, lo que puede ser hecho, lo que puede ser dicho) para que, en conjunto, se aprendan las ideas fundamentales de las ciencias gracias a una coherencia entre estas diferentes dimensiones. Y esto se puede conseguir si la clase se presenta como un discurso transformador de las ideas, de las acciones y de los lenguajes (Halliday et al., 1993).

Teniendo en cuenta el carácter sistémico de la clase, este discurso ha de ser mantenido por la interacción entre el alumnado, el profesorado y el conocimiento de los libros y las lecciones (Fig.1) Puesto que el texto (el libro, la información por internet, el audiovisual) es imprescindible, una de las preocupaciones principales del profesorado es darle vida, convertirlo en «experiencia» del alumnado y por esto el discurso requiere la mediación de diversos modos comunicativos ${ }^{5}$ (Jorba, 1998; Rowell, 1998; Sutton, 1996).

El lenguaje es central. El problema es que puede confundirse con las ideas y suscitar la creencia de que, si es riguroso, reproduce una verdad que existe objetivamente.

El reto es conseguir que los alumnos aprendan a «hablar y escribir ciencias» y para ello han de superar el mito de la «suprema bondad del lenguaje especializado» para descubrir que el lenguaje es un juego con 
el que pueden generar una realidad pensable sobre la cual pueden actuar, de acuerdo con una intención.

En realidad, han de descubrir que no existe el lenguaje especializado, sino sólo el lenguaje.

Una teoría sobre los contenidos escolares ha de tener en cuenta la función crucial del lenguaje, puesto que el conocimiento no está en la naturaleza (que no es un libro) sino en nuestra interacción con ella (Vygotsky, 1934, 1985).

Es necesario un concepto de ciencia compatible con lo que se ha venido diciendo hasta ahora.

\section{Nuevos modelos de ciencia: intervención, actividad científica «realista» pero de «racionalidad mode- rada», epistemología fundamentada en valores «no cientistas»}

La revolución cognitiva y la sociolingüística no han dejado incólume el concepto de ciencia. La filosofía de la ciencia ha participado activamente en los debates promovidos por las ciencias cognitivas (Hacking, 1983). Podemos ahora comprender mejor lo que es el conocimiento científico y la participación que tiene en su configuración la finalidad de «tener que enseñarlo».

Nos interesa especialmente la concepción semántica de teoría científica, que, junto con el realismo «perspectivista» y la «racionalidad hipotética» (Giere, 1988), inspira un modelo de «ciencia en la escuela», el cual resalta que lo más importante es enseñar a pensar mediante teorías que se vinculan a los fenómenos como consecuencia de una acción humana transformadora, que se comunica con un lenguaje propio.

Según Giere (1988), podemos identificar un «aspecto sintáctico» de las teorías, es decir, el cuerpo de teoremas enunciados en el lenguaje particular elegido para expresar esta teoría; y un «aspecto semántico», formado por el conjunto de modelos que le dan significado. Si se tiene en cuenta sólo el primer aspecto, puede parecer que el lenguaje «es» la teoría; pero si se consideran a la vez los dos, el lenguaje usado para expresar la teoría no es lo fundamental, sino que los modelos (los hechos del mundo que son sus ejemplos) ocupan el primer plano. Veamos el ejemplo que proporciona el propio Giere: los osciladores armónicos lineales pueden representarse por un conjunto de ecuaciones; pero pueden representarse también por el péndulo simple, por el muelle, por los amortiguadores del automóbil... es decir, por un conglomerado de modelos con diversos grados de especificidad, de los cuales se podría hablar de otra manera y no únicamente con los lenguajes matemáticos que son consecuencia de la reconstrucción lógica de los experimentos (Fig. 3).

Así, las teorías científicas tienen significado en relación con los fenómenos en los que se ha intervenido (son «experiencia», no sólo experimento) y por esto Giere insiste en que una teoría es un conjunto de modelos y de hechos del mundo que se reconocen «similares» a los modelos y que han sido interpretados por un proceso de modelización con sus propias «reglas del juego» o hipótesis teóricas. (Fig. 4)
Figura 3

Los modelos teóricos, según Giere (1988).

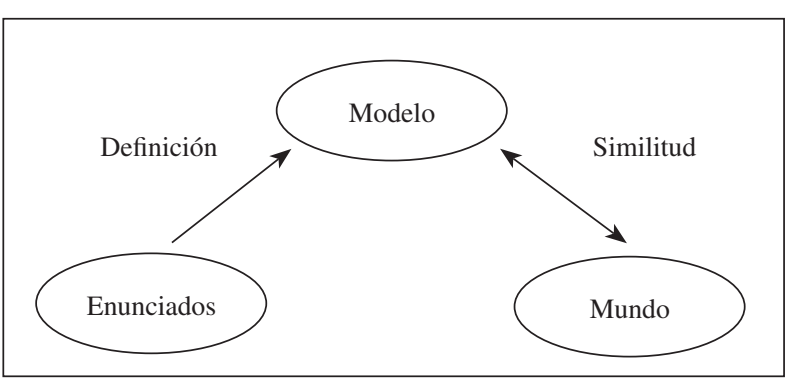

Figura 4

El proceso de modelización.

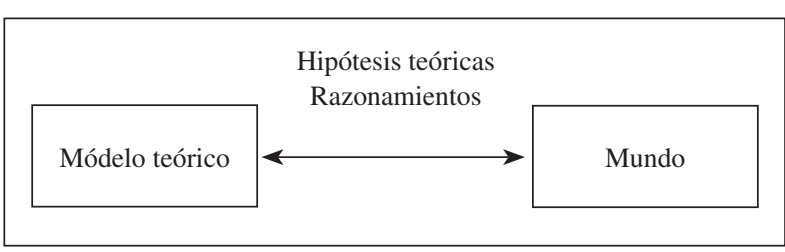

Las formulaciones lingüísticas de las teorías son verdaderas sólo si lo que pretenden y consiguen es caracterizar a la población de modelos. Por ello, en la ciencia escolar son válidos diversos modelos teóricos que «representan el mundo» con un diferente grado de abstracción, al igual que los enunciados que se usan para caracterizarlos.

Podríamos reconstruir la historia de la ciencia haciendo ver que ha habido diversidad de modelos y diversidad de lenguajes, y que todos ellos han intentado comunicar ideas y acciones sobre el mundo: todos ellos han «transformado el mundo» (Duschl, 1996). Quizás se podría reconstruir de la misma manera la «historia de la ciencia del alumno». Ya que ha de poder pensar, hablar y actuar para generar conocimientos, los modelos teóricos han de poder evolucionar y desarrollarse a lo largo de la enseñanza.

La principal aportación que puede hacer la ciencia a la educación de las personas es enseñar a pensar sobre los resultados de una intervención con motivo y objetivo, mediante «modelos» que integren reglas de actuación y lenguajes con los cuales se socialicen los conocimientos.

Las acciones del alumnado en la clase de ciencias adquieren significado científico al llevarse a cabo intencionadamente, en el marco de un modelo científico. De esta manera forman parte de una «actividad científica escolar» por la cual se transforma la manera de mirar gracias a la generación de los lenguajes adecuados; es decir, se genera conocimiento. 


\section{A modo de síntesis}

- Una teoría de los contenidos escolares ha de conseguir que el alumnado vea la clase de ciencias como una ocasión de entrar en una historia (de vivir la historia de «su» ciencia) como protagonistas, como narradores y como guionistas. Ha de tener en cuenta lo que se sabe del pensamiento individual, la manera de compartirlo mediante el lenguaje y la cultura científica que comparten quienes juegan «el juego de la ciencia».

Esta historia de la ciencia de cada alumno se vive al «hacer algo» de lo que sepa hablar y que le introduzca al planteamiento de problemas y a la búsqueda de estrategias de resolución, a la realización de investigaciones, y se inicia al disponer de una «buena pregunta»... que se ha de aprender a formular.

Ha de conducir la construcción de los hechos científicos propios del currículo, gracias a la combinación de acciones y de pensamientos en el marco de un «modelo», para que «aprender ciencias» sea transformar sus representaciones del mundo.

- Una teoría de los contenidos escolares ha de proporcionar criterios para no confundir el «conocimiento» con la letra inerte de los textos (sean cuales sean sus formatos) y para combatir el cientificismo y el enciclopedismo.

Por ello se ha de aceptar que:

- La capacidad de conocer es una de las características más notables de todas las personas, que «hace feliz» cuando es sabia porque se sabe aplicar.

- No todos los conocimientos tienen la misma calidad educativa (Gardner, 2000).
- Las vías de acceso al conocimiento son muchas, pero siempre han de acoplarse las tres dimensiones de la cognición: hacer, pensar, comunicar.

A partir de aquí llegamos al meollo del problema. ¿Qué pueden hacer tan significativamente los alumnos de ciencias de manera que puedan hablar de ello, si aún les faltan las palabras científicas que han transformado el mundo de los fenómenos en un mundo pensable gracias a una teoría y una práctica a la que ellos no pueden acceder?

\section{DEL «QUÉ ENSEÑAR» A UNA «TEORÍA DE LOS CONTENIDOS»O «CIENCIA ESCOLAR»}

Después de todo lo que se ha ido diciendo, voy a referirme a los «temas» de la «ciencia escolar», puesto que los conocimientos (¡dejemos ya de hablar de contenidos!) sólo lo son si sustentan una actividad científica escolar, motivada y con una finalidad, que se concreta en propuestas docentes temáticas coherentes e interesantes.

Para concretar una propuesta de «temas para la ciencia escolar» se han de tomar decisiones que no podemos tratar aquí. Lo que sí se puede hacer ahora es una propuesta para la organización de la actividad científica escolar: ésta será mi aportación a un esbozo de la «teoría de los contenidos».

\section{Los elementos de la teoría}

Empezaré por retomar las propuestas de White sobre las «dimensiones de los contenidos» (1994), mostrando su correlación con las propuestas teóricas que se han ido haciendo hasta aquí (Tabla I).

Tabla I

Las dimensiones de los contenidos que pueden ser desarrolladas a partir de aportaciones actuales de la didáctica de las ciencias.

\begin{tabular}{|c|c|}
\hline Dimensiones de los contenidos (White, 1994) & Aportaciones actuales de la DC \\
\hline Obertura a la experiencia común & Contar con las ideas previas \\
\hline Abstracción & Organizar la actividad escolar a partir de los modelos teóricos o las ideas estructurantes \\
\hline Aceptar la presencia de palabras comunes & $\begin{array}{l}\text { Dar prioridad a la comunicación mediante el lenguaje cotidiano antes de introducir los } \\
\text { términos científicos }\end{array}$ \\
\hline $\begin{array}{l}\text { Tener en cuenta que los alumnos utilizan modelos } \\
\text { alternativos con poder explicativo }\end{array}$ & $\begin{array}{l}\text { Desarrollar argumentación científica en el aula para sustentar el proceso de modelización } \\
\text { científica }\end{array}$ \\
\hline Complejidad & Trabajar en las tres dimensiones: hacer, pensar, comunicar \\
\hline Han de poder emocionar & $\begin{array}{l}\text { Conectar con los motivos que hacen que los estudiantes quieran aprender y desarrollar } \\
\text { procesos de metacognición }\end{array}$ \\
\hline Han de ser demostrables, no arbitrarios & Utilizar un modelo de ciencia de realismo y racionalidad «moderados» \\
\hline Requieren aceptación social & Ha de conectar con una finalidad educativa que tenga consenso \\
\hline $\begin{array}{l}\text { Han de poder conectar con los otros } \\
\text { conocimientos que también se irán aprendiendo }\end{array}$ & Se han de estructurar las ideas básicas e irreductibles para continuar aprendiendo \\
\hline Siempre son mezcla de diferentes conocimientos & Tener en cuenta las inteligencias múltiples y diseñar diferentes vías de acceso y de progresión \\
\hline
\end{tabular}


Como vemos, la ciencia escolar se extiende en un espacio cuyas dimensiones son diferentes a las de las disciplinas científicas clásicas: sus raíces están aún en la experiencia y el lenguaje comunes pero tiende a la abstracción simplificadora con consenso social y con una finalidad educativa; la demostrabilidad de sus principios ha de ganarse mediante itinerarios personales. Por esto no puede ser, ahora, un calco de ninguna de las disciplinas científicas que se enseñan en la universidad y en las cuales se han formado los profesores, pero tampoco puede obviarlas ni desconocerlas ${ }^{6}$. Para ello, tal como indica García (1998, 2001), es necesario «conocimiento metadisciplinar»: saber metacientífico, cosmovisiones ideológicas que orientan sistemas de ideas para tomar decisiones.

Con seriedad y rigor, los temas deberían ordenarse alrededor de los «modelos» básicos e irreductibles (Izquierdo, 1994; Izquierdo, Solsona y Cabello, 1994) que permiten interpretar conjuntos de fenómenos que son relevantes para la formación de todas las personas, los cuales se han de identificar, seleccionar y reelaborar (Black, 1986), Y, tal como se desprende de González (2001), estos modelos básicos no son ni los más modernos ni los más antiguos; son los que agrupan las ideas que fundamentan los conocimientos derivados de una determinada intencionalidad educativa que incluye enseñar a razonar a la manera de las ciencias físicas y naturales.

A partir de la tabla I podemos establecer los elementos de la teoría de los contenidos, es decir, lo que se ha de tener en cuenta para escoger los temas de la ciencia escolar, que se relacionan entre sí y se condicionan unos a otros. Estos elementos del «qué enseñar» que se proponen a continuación nos resultan familiares; pero creo que, a partir de lo que se ha ido reflexionando hasta aquí, toman un nuevo sentido:

- Los objetivos o finalidades educativas, que proporcionan el énfasis que caracteriza a la propuesta.

- Los núcleos temáticos, organizados alrededor de los modelos teóricos apropiados, con sus vías de acceso y sus conocimientos estructurantes.

- Los procesos de justificación, con sus estrategias de progresión y sus episodios y hechos paradigmáticos.

- Los criterios para conectar con otros conocimientos, reconocer los conocimientos nuevos que pueden conectarse y los que han de dejarse para otro momento.

Veámoslo con más detalle.

\section{Objetivos}

Condicionan todo lo que va a venir a continuación. Algunos de ellos son optativos, según lo que se quiera o se pueda conseguir (un currículo más o menos humanista, más o menos tecnológico, más o menos cotidiano...) y otros son irrenunciables, porque se refieren a valores educativos básicos: democracia (no sexismo, respeto a la diversidad..), educar para un intervención respetuosa con el medio, ofrecer oportunidades para razonar, la alfabetización científica para todos, quizás la employability...

\section{«Núcleos temáticos»}

Han de ser coherentes con los objetivos. Han de tener en cuenta las vías de acceso, que pueden ser diversas incluso para un mismo tema con el fin de conseguir motivar a los alumnos. Han de vincularse a alguno de los modelos teóricos que son indispensables porque no se pueden reducir unos a otros y porque de ellos se derivan los conocimientos estructurantes que dan sentido a conceptos como energía, equilibrio, estructura, cambio... que toman su propio sentido (se miden de manera diferente) en cada modelo teórico científico.

En lo que se refiere estrictamente al qué enseñar (que es lo que ahora nos ocupa), parece razonable que los núcleos temáticos se desarrollen de la manera siguiente:

- Una narración inicial que introduce el tema en el contexto adecuado.

- De ella se desprenden episodios que dan lugar a un estudio más profundo, el cual incluye la evidencia experimental.

- Con ello se construyen hechos paradigmáticos, que son los fenómenos que pueden explicarse mediante las entidades que se han introducido en el proceso de modelización y que constituyen un ejemplar que tiene la función de «modelo» para interpretar otros fenómenos similares.

\section{Procesos epistemológicos}

Se ha de prever lo que se ha de saber hacer, lo que se ha de saber «escribir», representar, decir, los problemas que se llegarán a plantear y los que se pueden resolver para proponer una estrategia de progresión, que dé ritmo al aprendizaje (por ejemplo, estableciendo un «ciclo de aprendizaje» con diferentes etapas: explorar, introducir, aplicar).

\section{Criterios}

Para estructurar los conocimientos, se ha de poder reconocer cuando los conocimientos son incompatibles o irrelevantes y cómo conectar con otros conocimientos, para poder continuar aprendiendo, para seleccionar más información relevante y rechazar la que no lo es.

Una vez diseñada una propuesta concreta, ha de tener también sus nuevos libros fundacionales, sus nuevos rituales de ciencia escolar según sus finalidades propias. 
Los «contenidos» deberían verse como una historia con diferentes episodios y hechos, en la cual fenómenos del mundo que parecen desconectados toman sentido al relacionarse entre sí mediante entidades científicas de las que se tienen evidencias experimentales que se pueden razonar.

Es una historia inacabada, que continúa desarrollándose en diferentes contextos y en la que se puede participar de maneras diferentes a lo largo de toda la vida.

La DC debería fundamentar con valentía nuevas propuestas de temas para la ciencia escolar adecuadas a las diversas audiencias, que ya no son los antiguos candidatos a las disciplinas universitarias de siempre.

\section{Hacia nuevos currículos}

Hay muchas propuestas para cambiar los programas de ciencias y para substituir los temas tradicionales por otros que conecten mejor con lo cotidiano o se refieran a las interacciones entre ciencia, tecnología y sociedad. Incluso los programas oficiales muestran un cierto enfoque transdisciplinar y, aparentemente, pretenden la implicación personal de los estudiantes; pero estos cambios no se consolidan, aún no llegan a la escuela.

Y no sólo esto: aparecen centros para el aprendizaje no formal, más allá de la escuela; se ofrecen recursos diversos por internet; los museos de ciencias reciben subvenciones millonarias y deslumbran al ciudadano con las maravillas científicas que en ellos se muestran. Esta enorme y novedosa oferta de conocimientos es fantástica, pero su dispersión puede hacerla inútil para la educación.

Podemos avanzar a ciegas por este nuevo panorama o bien orientarnos mediante los conocimientos que la revolución cognitiva, la nueva sociolingüística y la filosofía de la ciencia nos han proporcionado, cribados y reconstruidos por el conocimiento profesional (didáctico) de los profesores. Las aportaciones que se han hecho en el apartado anterior permiten diseñar propuestas que funcionen en un doble sentido: organizar los contenidos de los temas básicos para que se puedan aprender dando lugar a una gran diversidad de currículos; y permitir su futura diversificación personal al continuar aprendiendo a partir de ofertas externas a la escuela.

Lo más comprometido es identificar los modelos teóricos que dan consistencia a los núcleos temáticos, mostrando cómo intervenir en ellos. Si esto se hiciera bien y la identificación fuera compartida por los profesores, por los divulgadores científicos, por los diseñadores de museos y de web sería posible que propuestas diferentes compartieran los mismos criterios y incidieran en los mismos procesos epistemológicos, contribuyendo así al aprendizaje, es decir, al desarrollo de la historia de la ciencia de cada uno.
El MT genera las entidades conceptuales que se prevén en el currículo mediante un proceso de modelización (Fig. 4), no a la inversa. El modelo se vincula con los hechos concretos (hechos paradigmáticos o ejemplares) en los que se pueda intervenir, adecuados a las posibilidades de formular en clase «hipótesis teóricas» que se puedan contrastar experimentalmente y que sean apropiadas a las diferentes entidades científicas que se han de introducir. Estas entidades permiten poder hablar y escribir sobre los fenómenos mediante el lenguaje apropiado.

Veamos un ejemplo referente a la enseñanza de la química. Habitualmente ésta empieza por la caracterización o definición de los conceptos químicos fundamentales, como substancia, elemento, átomo... Pero de esta manera no se accede a lo esencial de la química, que es la visión del mundo que proporciona mediante lo que podemos llamar modelo de cambio químico (MCQ), con lo cual se identifican determinados fenómenos y se caracterizan por su «similitud química». El MCQ establece las «reglas del juego» al hablar y escribir sobre lo que pasa cuando se interviene en este tipos de cambio; limita lo que se puede hacer al intervenir en él y, a la vez, establece lo que es y lo que no es este tipo de cambio. Con ello, van siendo necesarios los conceptos químicos, que adquieren significado en el juego de controlar el cambio químico. En efecto, las reglas de juego se recuerdan mejor en términos de algunas entidades (átomos, moléculas, enlaces) de las que se derivan los conceptos y términos específicos, que adquieren sentido sólo al final del proceso de modelización (por ejemplo, no se puede comprender bien lo que es una substancia química al margen del MCQ).

Como contrapartida a esta selección de contenidos en función de un MT, se han de eliminar los conocimientos incompatibles o irrelevantes que pertenencen a otro modelo. Sólo se van a poder conectar significativamente conocimientos que pertenezcan al mismo MT y, aunque esto ya es una gran ayuda para conectar informaciones, un determinado modelo no sirve para cualquier fenómeno; los puentes entre ellos necesitan de un aprendizaje específico.

En la escuela debería planificarse un currículo coordinado, sin repeticiones y completo (los diferentes profesores saben lo que es irrenunciable en su asignatura, pero en conjunto se deberá renunciar a lo que no es imprescindible), con pocas ideas y bien relacionadas; los hechos que sean sus ejemplos y que, a la vez, permitan actividad relevante y autónoma, sobre la que se pueda hablar y representar con diversidad de lenguajes que funcionen como «signos», contando con la implicación personal.

La divulgación científica debería tener en cuenta estas ideas básicas y ayudar al público a conectar con ellas las informaciones diversas que se le proporcionan. 


\section{CONCLUSIONES}

Los conocimientos escolares son parte de un sistema didáctico y sería muy necesario disponer de criterios para seleccionarlos de manera adecuada. Disponemos ahora de suficiente investigación en didáctica de las ciencias para empezar a enfocar el problema que se presenta debido a la permanencia de contenidos tradicionales que no permiten que el sistema didáctico funcione de la manera adecuada o de nuevos contenidos que se presentan sin orden ni concierto.

La clase tradicional se centraba en los libros de texto hasta el punto de confundir los conocimientos científicos escolares con el contenido del libro; la clase actual ha de centrarse en la creación de lenguaje «honesto», con el cual se piensa y se actúa, para poder comprender lo que es en realidad el lenguaje científico. (El lenguaje, liberado del requisito de ser «preciso» para pasar a ser «honesto» ${ }^{7}$. Una vez superada la exigencia de precisión del lenguaje, por imposible, se puede «jugar con el lenguaje» para favorecer la interpretación más válida de un término de significado impreciso o demasiado amplio, pero no necesariamente erróneo del todo.)

Así, el primer paso es convencernos de que los «contenidos» no están en ninguna parte, ni en un libro ni en la red. Son «conocimiento» y son inseparables de la actividad científica. Por ello una teoría de los contenidos (escolares) es indisociable de la finalidad de promover una actividad científiica (escolar o ciudadana).

Las disciplinas escolares ya no se identifican con las clásicas, pero éstas también han evolucionado e incluso van desapareciendo de la universidad. ¿Significa esto que están apareciendo nuevas disciplinas que son sólo escolares? No tengo una respuesta clara a esta pregunta; lo que sí que va quedando claro es que la transposición didáctica va a ser más radical a medida que el diseño de los contenidos «aprendibles» vaya adquiriendo la autonomía que le corresponde; no se trata de adaptar las ideas de los científicos a la clase, sino de diseñar unas ciencias propias de la clase, adecuadas a las ideas y sentimientos de los alumnos, que tengan en cuenta los obstáculos que se van a presentar, pero que sepan aprovechar lo que el alumnado ya conoce y hacia lo cual está motivado.
Todo lo que se ha dicho hasta aquí plantea un interesante trabajo a emprender: establecer las reglas de juego para diseñar conocimientos que unas personas determinadas puedan aprender. Si se consigue avanzar en esta línea, podremos ser muy útiles: en educación a distancia, contribuyendo a que los museos de ciencias sean más eficaces, elaborando nuevos materiales didácticos, utilizando las TIC de manera adecuada, ajustando mejor los requisitos para la promoción de nivel educativo... Podemos contribuir a no desanimar al público, que es lo que ocurre cuando se ofrecen conocimientos que parecen sencillos, pero que en realidad casi nadie llega a dominar con autonomía.

\section{NOTAS}

* Ponencia presentada en el VI Congreso Internacional sobre Investigación en la Didáctica de las Ciencias (Barcelona, 12 al 15 de septiembre de 2001). ${ }^{1}$ Es uno de los componentes del currículo; se ha de concretar además cómo y cuándo enseñar y cómo evaluar, pero no me refiero a ello en este momento.

${ }^{2}$ Aunque quizás en el futuro no sea el único ni el más importante en el que se pueda enseñar-aprender.

${ }^{3}$ En Kiel, en la III Conferencia bianual de ESERA.

${ }^{4}$ Recientemente se ha enriquecido esta reflexión con ideas pocedentes del «paradigma de la complejidad», Investigación en la Escuela.

${ }^{5}$ Diversas investigaciones recientes se centran en el análisis de la comunicación multimodal en las clases de ciencias (Márquez et al., 2003).

${ }^{6}$ Con palabras de García, por ejemplo, afirmaciones simplistas como «la intervención del hombre lleva a la destrucción de los ecosistemas, la contaminación provoca graves perturbaciones del clima, rompe los ciclos naturales...», si no se profundiza en lo que son estos ciclos naturales y en cómo funcionan, no permiten ir muy lejos en la formación de los futuros científicos, porque inducen a pensar que la dinámica de la naturaleza depende de las personas y no del sol.

${ }^{7}$ Entiendo por lenguaje deshonesto el del parte metereológico: miles de personas lo «leen» en la TV, diariamente, pero cuántas podrían explicar el significado de las líneas que allí aparecen. Más o menos se sabe que si hay una $\mathrm{D}$ encima de nuestra ciudad, será que lloverá... o incluso alguien podrá relacionar la estructura del conjunto de líneas «isóbaras» con un posible vendaval... pero esto no tiene nada que ver con comprender el lenguaje simbólico que allí aparece. Lo «honesto» sería, o bien explicar bien lo que pasa, o utilizar la simbología adecuada a las conclusiones que finalmente van a retener y comprender los televidentes. 


\section{REFERENCIAS BIBLIOGRÁFICAS}

BLACK, P. (1986). Integrated or co-ordinated science? School Science Review, 67(241), pp. 669-681.

CHEVALLARD, Y. (1991). La transposición didáctica. Buenos Aires: Aique.

CHOMSKY, N. (1980). Rules and Representations. Nueva York: Academic Press.

DUSCHL, R.A. (1996). Renovar la enseñanza de las ciencias. Importancia de las teorías y su desarrollo. Madrid: Narcea.

ECO, H. (1999). Kant i l'ornitorrinc. Barcelona: Destino.

GARCÍA DÍAZ, E. (1998). Hacia una teoría alternativa de los contenidos escolares. Sevilla: Díada

GARCÍA DÍAZ, E. (2001). De los problemas científicos a los problemas medioambientales. Alambique, 29, pp. 25-33.

GARDNER, H., KORNHABER, M. y WAKE, W. (1996). Intelligence, Multiple Perspectives. For Worth, Tex. Harcourt Brace.

GARDNER, H. (2000). La educación de la mente y el conocimiento de las disciplinas. Madrid: Paidós.

GONZÁLEZ, F. (2001). Biología para una nueva generación. Nuevos contenidos y nuevos continentes. Alambique, 29, pp. 63-69.

GUIDONI, P. (1985). On natural thinking. European Journal of Science Education, 7(2), pp. 133-140.

GIERE, R. (1988). Explaining Science. A Cogntive Approach. Chicago: University of Chicago Press.

GUNDEM, B. (2000). Undersatnding European Didactics, en Moon et al. (eds.). Pouledge International Companion to Education, pp. 235-262. Londres: Routledge.

FENSHAM, P. (2001). Science content as problematic. Issues for research, en Behrendt, H. et al. (eds.). Research in Science education: Past, present and future, pp. 27-42. Dordrecht: Kluwer

FENSHAM, P. (2004). Focus on Content, en «Defining an Identity», pp. 145-161. Dordrecht: Kluwer.

HACKING, I. (1983). Representing and Intervening: Introductory Topics in the Philosophy of Natural Science. Cambridge: Cambridge University Press.

HALLIDAY, M.A.K. y MARTÍN, J.R. (1993). Writing Science: Literacy and discursive power. Londres: Falmer Press.

HOPMANN, S. y RIQUARTS, K. (1995). Didaktik and/or Curriculum. Kiel: IPN

IZQUIERDO, M., SANMARTÍ, N. y ESPINET, M. (1999). Fundamentación y diseño de las prácticas escolares de ciencias experimentales. Enseñanza de las Ciencias, 17(1), pp. 45-59.

IZQUIERDO, M., SANMARTÍ, N., ESPINET, M., GARCÍA, M.P. y PUJOL, R.M. (1999). Caracterización y fundamentación de la ciencia escolar: Enseñanza de las Ciencias, núm. extra, pp. 79-92.
IZQUIERDO, M., SANMARTÍ, N., ESPINET, M. y GARCÍA, P. (2000). Application of a model shift of scientific knowledge: From the metaphor of the «book» to the metaphor of «discourse». Comunicación presentada en la 25 Conferencia Internacional de la ATEE. Barcelona.

IZQUIERDO, M. (1994). Las ciencias de la naturaleza de la ESO, ¿un área común o disciplinas distintas? Infancia y Aprendizaje, 63, pp. 31-34.

IZQUIERDO, M., SOLSONA, N. y CABELLO, M. (1994). Proyecto Ciencias, 12-16. Alambique 1, pp. 61-74.

JORBA, J. (1998). La comunicació i les habilitats cognitivolingüístiques, en Jorba, J. et al. (eds.). Parlar $i$ escriure per aprendre. Barcelona: ICE-UAB.

KLAFKI, W. (1958). Didaktische Analyse als Kern der Unterrichtsvorbereitung. Basel: Wienheim.

KUHN, D. (1993). Science as argument; implications for teaching and learning scientific thinking. Science Education, 77(3), pp. 319-337.

LAKOFF, G. y JOHNSON, M. (1995, 1980). Metáforas de la vida cotidiana. Madrid: Cátedra.

LEMKE, J. L. (1997). Aprender a hablar ciencia. Barcelona: Paidós.

MÁRQUEZ, C., IZQUIERDO, M. y ESPINET, M. (2003). Comunicación multimodal en la clase de ciencias: el ciclo del agua. Enseñanza de las Ciencias, pp. 371-386.

MILLAR, R. y DRIVER, R. (1987). Beyond processes. Studies in Science Education, 14, pp. 33-62.

OHLSSON, S. (1995). Learning to do and learning to understand. A lesson and a challenge for cognitive modelling, en Reimann, P. Learning in humans and machines. Nueva York: Pergamon.

PRO, DE, A. y SAURA, O. (2001). Nuevos tiempos, nuevos contenidos. Alambique, 29, pp. 63-69.

ROWELL, P. A. (1998). Learning in school science: The promises and practices of writing. Studies in Science Education, 30, pp. 19-56.

RUE, J. (2002). Qué enseñar y por qué. Barcelona: Paidós

SHULMAN, L. (1995). Wisdom for practice and wisdom of practice. Two aspects of a didactics of substance, en Hopmann, S. y Riquarts, K. (eds). Didaktik and/ or Curriculum. Kile: IPN

SKINNER, B.F. (1968). The Technology of Teaching. Nueva York: Apleton Century Crofts.

SUTTON, C.R. (1996). Beliefs about science and beliefs about language. Int. J. Sci. Educ., 18(1), pp. 1-18.

VYGOTSKY, L.S. (1985). Pensamiento y lenguaje. Buenos Aires: La Pléyade.

WHITE, R. (1994). The dimensions of Content, en «The content of Science».

WITTGENSTEIN, L. (1997). Investigacions filosòfiques. Barcelona: Edicions 62 (1953). 